\title{
Extracting Dynamical Equations from Experimental Data is NP Hard
}

\author{
Toby S. Cubitt, ${ }^{1}$ Jens Eisert, ${ }^{2}$ and Michael M. Wolf ${ }^{3}$ \\ ${ }^{1}$ Departamento de Análisis Matemático, Universidad Complutense de Madrid, Plaza de Ciencias 3, \\ Ciudad Universitaria, 28040 Madrid, Spain \\ ${ }^{2}$ Dahlem Center for Complex Quantum Systems, Freie Universität Berlin, 14195 Berlin, Germany \\ ${ }^{3}$ Zentrum Mathematik, Technische Universität München, 85748 Garching, Germany
}

(Received 24 June 2011; published 22 March 2012)

\begin{abstract}
The behavior of any physical system is governed by its underlying dynamical equations. Much of physics is concerned with discovering these dynamical equations and understanding their consequences. In this Letter, we show that, remarkably, identifying the underlying dynamical equation from any amount of experimental data, however precise, is a provably computationally hard problem (it is NP hard), both for classical and quantum mechanical systems. As a by-product of this work, we give complexity-theoretic answers to both the quantum and classical embedding problems, two long-standing open problems in mathematics (the classical problem, in particular, dating back over 70 years).
\end{abstract}

DOI: 10.1103/PhysRevLett.108.120503

PACS numbers: 03.67.Ac, 03.65.Ud, 03.65.Yz, 89.70.Eg

A large part of physics is concerned with identifying the dynamical equations of physical systems and understanding their consequences. But how do we deduce the dynamical equations from experimental observations? Whether deducing the laws of celestial mechanics from observations of the planets, determining economic laws from observing monetary parameters, or deducing quantum mechanical equations from observations of atoms, this task is clearly a fundamental part of physics and, indeed, science in general. The task of identifying dynamical equations from experimental data also turns out to be closely related, in both the classical and quantum mechanical cases, to long-standing open problems in mathematics (in the classical case, dating back to 1937 [1]).

In this Letter, we give complexity-theoretic solutions to both these open problems. And these results lead to a surprising conclusion: Regardless of how much information one obtains through measuring a system, extracting the underlying dynamical equations from those measurement data is, in general, an intractable problem. More precisely, it is NP hard. This means that any computationally efficient method of determining which dynamical equations are consistent with a set of measurement data would solve the (in)famous $\mathrm{P}$ versus NP problem [2], by implying that $\mathrm{P}=\mathrm{NP}$. Thus, if $\mathrm{P} \neq \mathrm{NP}$, as is widely believed, there cannot exist an efficient method of deducing dynamical equations from any amount of experimental data. We also prove the other direction: By reducing to an $\mathrm{NP}$-complete problem we show that, if $\mathrm{P}=\mathrm{NP}$, then there does exist an efficient algorithm for extracting dynamical equations from experimental data. Thus, the question of whether there exists an efficient method for determining dynamical equations from measurement data is equivalent to the $\mathrm{P}$ versus NP question.

Note that we are not restricting ourselves here to fundamental theories, where other theoretical considerations may impose simplifications on the desired form of the equations. We are also considering effective dynamical equations, as encountered in the majority of experiments, where the full range of possible dynamical equations can, in principle, be observed.

In the classical setting, the problem of extracting dynamical models from experimental data has spawned an entire field known as system identification [3], which forms part of control engineering - after all, the precise knowledge of the dissipation is crucial for actually understanding what control steps to apply. In the quantum case, interest in understanding quantum dynamics, especially externally induced noise and decoherence, has been spurred on by efforts to develop quantum information processing technology $[4,5]$. Indeed, the primary goal of many experiments is precisely to characterize and understand the dynamics of a specific quantum system [6-10]. This is precisely the task that we show to be computationally intractable in general (assuming $\mathrm{P} \neq \mathrm{NP}$ ), both in quantum mechanics and in classical physics.

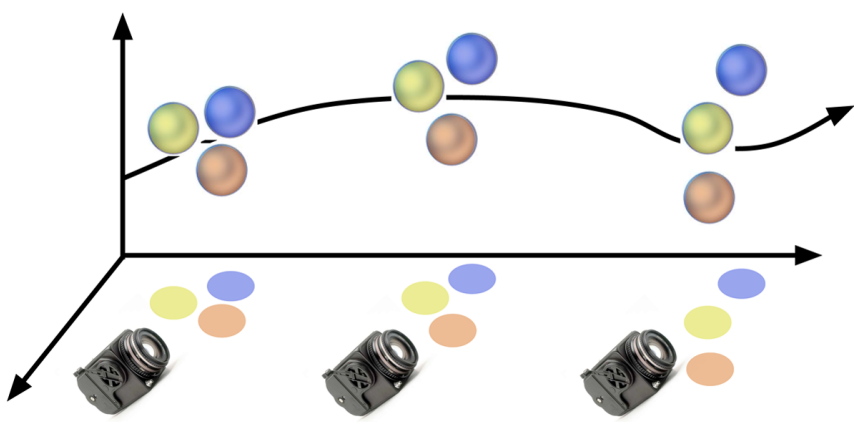

FIG. 1 (color online). In an experiment, we can gather snapshots of the state of a physical system at various points in time. To understand the physics behind the system's behavior, we must reconstruct the underlying dynamical equations from the snapshots. 
Results.-Let us make the task more concrete. We will throughout consider open system dynamics, which takes external influences and noise into account. Recall that in classical mechanics, the most general state of a system is described by a probability distribution $p$ over its state space, which for simplicity we will take to be finite dimensional. Its evolution is then described by a master equation, whose form is determined by the system's Liouvillian, corresponding to a matrix $L$, as $\dot{p}=L p$. The Liouvillian expresses interactions, conservation laws, external noise, etc.; in short, it describes the underlying physics. In order for the probabilities to remain positive and sum to one, the elements $L_{i, j}$ must obey two simple conditions [11]: (i) $L_{i \neq j} \geq 0$ and (ii) $\sum_{i} L_{i, j}=0$.

In the quantum setting, the density matrix $\rho$ plays the analogous role to that of the classical probability distribution, but the quantum master equations are still determined by a Liouvillian:

$$
\dot{\rho}=\mathcal{L}(\rho) .
$$

In his seminal 1976 paper [12], Lindblad established the general form that any quantum Liouvillian must take if it is to generate a completely positive trace-preserving evolution (so that density matrices always evolve into density matrices, directly analogous to probabilities remaining positive and normalized in the classical case):

$$
\mathcal{L}(\rho)=i[\rho, H]+\sum_{\alpha, \beta} G_{\alpha, \beta}\left(F_{\alpha} \rho F_{\beta}^{\dagger}-\frac{1}{2}\left\{F_{\beta}^{\dagger} F_{\alpha}, \rho\right\}_{+}\right) .
$$

Here, $H$ is the Hamiltonian of the system, $G$ is a positive semidefinite matrix and, along with the matrices $F_{\alpha}$, describes decoherence processes. ([.,.] and $\{., .\}_{+}$denote, respectively, the commutator and anticommutator.) These master equations of the Lindblad form have become the mainstay of the dynamical theory of open quantum systems, and are crucial to the description of quantum mechanics experiments [13]. In principle, the Liouvillian could itself be time dependent, describing a system whose underlying physics is changing over time. Here, we restrict our attention to the problem of finding a time-independent Liouvillian, as this is a good assumption for experiments in which external parameters are held constant. The more general time-dependent problem is expected to be harder still.

What is the best possible data that an experimentalist can conceivably gather about an evolving system? At least in principle, they can repeatedly prepare the system in any chosen initial state, allow it to evolve for some period of time, and then perform any measurement. In fact, for a careful choice of initial states and measurements, it is possible in this way to reconstruct a complete "snapshot" of the dynamics at any particular time (Fig. 1). In the quantum setting, this technique is known as quantum process tomography [5]. Quantum process tomography is now routinely carried out in many different physical systems, from NMR [6,7] to trapped ions [8], and from photons [9] to solid-state devices [10].

A tomographic snapshot tells us everything there is to know about the evolution at the time $t$ when the snapshot was taken. Each snapshot is a dynamical map $\mathcal{E}_{t}$, which describes how the initial state, $p_{0}$ or $\rho_{0}$, is transformed into $p(t)=\mathcal{E}_{t}\left(p_{0}\right)$ or $\rho(t)=\mathcal{E}_{t}\left(\rho_{0}\right)$. Any measurement at time $t$ can be viewed as an imperfect version of process tomography, giving partial information about the snapshot, and the outcome of any measurement of the system at time $t$ can be predicted once $\mathcal{E}_{t}$ is known. Thus, the most complete data that can be gathered about a system's dynamics consists of a set of snapshots taken at a sequence of different points in time.

Let us concentrate first on the quantum case. Quantum dynamical maps $\mathcal{E}_{t}$ are described mathematically by completely positive, trace-preserving (CPT) maps [5] (also known as quantum channels). The problem of deducing the dynamical equations from measurement data is then one of finding a Lindblad master equation (1) that accounts for the CPT snapshots $\mathcal{E}_{t}$. This is essentially the converse problem to that considered by Lindblad [12,14]. Given its relevance, it is not surprising that numerous heuristic numerical techniques have been applied to tackle this problem $[7,15]$. But unfortunately these give no guarantee as to whether a correct answer has been found. Our results show that the failure of these heuristic techniques is an inevitable consequence of the inherent intractability of the problem.

Before tackling the problem of finding dynamical equations, let us start by considering an apparently much simpler question: Given a single snapshot $\mathcal{E}$, does there even exist a Liouvillian $\mathcal{L}$ that could have generated it? Not every CPT map $\mathcal{E}$ can be generated by a Lindblad master equation $[16,17]$, so the question of the existence of such a Liouvillian [Eq. (2)] is a well-posed problem. A dynamical map that is generated by a Lindblad form Liouvillian is said to be Markovian, so this problem is sometimes referred to as the Markovianity problem. Non-Markovian snapshots [18] can arise if the environment carries a memory of the past, so that the system's evolution cannot be described by Eq. (1) in the first place, as that assumes the system is sufficiently isolated from its environment for its dynamics to be described independently.

It is important to note that, for the results to apply to real experimental data, we must take into account the fact that a snapshot can only ever be measured up to some experimental error. We should therefore be satisfied if we can answer the question for some approximation $\mathcal{E}^{\prime}$ to the measured snapshot $\mathcal{E}$, as long as the approximation is accurate up to the experimental error. Mathematically, this is known as a weak membership formulation of the problem.

To address the Markovianity problem, we will require some basic concepts from complexity theory. Recall that $\mathrm{P}$ is the class of computational problems that can be solved 
efficiently on a classical computer. The class NP instead only requires an efficient verification of solutions, and contains problems that are believed to be impossible to solve efficiently, such as the famous 3SAT problem, and the traveling salesman problem. A problem is NP hard if solving it efficiently would also lead to efficient solutions to all other NP problems. A problem that is both NP hard and is also itself in the class NP is said to be NP complete. The 3SAT and traveling salesman problems are both examples of NP-complete problems, whereas the problem of factoring large integers is an example of an NP problem that is believed not to be NP hard [19].

Rather than considering 3SAT, it is more convenient here to consider the equivalent 1-in-3SAT problem, into which 3SAT can easily be transformed [19], and which is therefore also NP complete. We will show that any instance of the 1-in-3SAT problem can be efficiently transformed into an instance of the Markovianity problem (see also $[20,21])$, thus proving that the latter is at least as hard as 1-in-3SAT; any efficient procedure for determining whether a snapshot has some underlying Liouvillian would immediately imply an efficient procedure for solving 1-in3SAT. But 1-in-3SAT is NP complete, so this would immediately give an efficient algorithm for solving any NP problem, implying $\mathrm{P}=\mathrm{NP}$. However, as discussed above, the Markovianity problem is just a special case of the more general-and more important-problem of extracting the underlying dynamical equations from experimental data. If $\mathrm{P} \neq \mathrm{NP}$, as is widely believed, then there cannot exist a computationally efficient method of deducing dynamical equations from any amount of experimental data.

We can go further than this. Through the relation to NPcomplete problems such as 1-in-3SAT, we can reduce the Markovianity problem to the task of solving an NPcomplete problem. This gives the first rigorous, provably correct algorithm for extracting the underlying dynamical equations from a set of experimental data, albeit one that is necessarily inefficient for systems with more than a few degrees of freedom (otherwise we would have proven $P=\mathrm{NP})$.

We have focused so far on the more complex case of quantum systems, and one might perhaps expect that systems governed by classical physics would be easier to analyze. However, essentially the same argument proves that exactly the same results hold for classical systems, too. (See also [20,21].)

The technical argument.- It is convenient to represent a snapshot $\mathcal{E}$ of the dynamics of a quantum system (a CPT map) by a matrix $E$,

$$
E_{i, j ; k, l}=\operatorname{Tr}[\mathcal{E}(|i\rangle\langle j|)|k\rangle\langle l|]
$$

(the row and column indices of $E$ are the double indices $i, j$ and $k, l$, respectively). Looked at this way, each measurement that is performed pins down the values of some of these matrix elements [5]. A snapshot of a Markovian evolution is then one with a Liouvillian $\mathcal{L}$ (represented in the same way by a matrix $L$ ) such that $E=e^{L}$, and, for all times $t \geq 0, E_{t}=e^{L t}$ are also valid quantum dynamical (CPT) maps.

The Markovianity problem can be transformed into an equivalent question about the Liouvillian. Inverting the relationship $E=e^{L}$, we have $L=\log E$. There are, however, infinitely many possible branches of the logarithm, since the phases of complex eigenvalues of $E$ are only defined modulo $2 \pi i$. The problem then becomes one of determining whether any one of these is a valid Liouvillian [i.e., in Lindblad form (2)]. This translates into the following necessary and sufficient conditions on the matrix $L$ [17]. (i) $L^{\Gamma}$ is Hermitian, where $\Gamma$ is defined by its action on basis elements: $|i, j\rangle\left\langle k,\left.l\right|^{\Gamma}=\mid i, k\right\rangle\langle j, l|$. (ii) $L$ fulfils the normalization $\langle\omega| L=0$, where $|\omega\rangle=\sum_{i}|i, i\rangle / \sqrt{d}$ is maximally entangled. (iii) $L$ satisfies the condition of complete positivity $(\mathrm{CCP})$, i.e., $(\mathbb{1}-\omega) L^{\Gamma}(\mathbb{1}-\omega) \geq 0$, $\omega=|\omega\rangle\langle\omega|$. All branches $L_{m}$ of the logarithm can be obtained by adding integer multiples of $2 \pi i$ to the eigenvalues of the principle branch $L_{0}$, so we can parametrize all the possible branches by a set of integers $m_{c}$ :

$$
\begin{gathered}
L_{m}=\log E=L_{0}+\sum_{c} m_{c} A^{(c)}, \\
A^{(c)}=2 \pi i\left[\left|l_{c}\right\rangle\left\langle r_{c}\right|-\mathbb{F}\left(\left|l_{c}\right\rangle\left\langle r_{c}\right|\right)\right],
\end{gathered}
$$

with $\left|l_{c}\right\rangle$ and $\left\langle r_{c}\right|$ the left and right eigenvectors of $E$. $\mathbb{F}$ is the operation $\mathbb{F}(|i, j\rangle\langle k, l|)=|j, i\rangle\left\langle l,\left.k\right|^{*}\right.$, where $*$ denotes the complex conjugate, and we have already restricted the parametrization to logarithms that satisfy condition (i).

We will prove that this Liouvillian problem is NP hard, by showing how to encode any instance of the NPcomplete 1-in-3SAT problem into it. Recall that the task in 1-in-3SAT is to determine whether a given logical expression can be satisfied or not. The expression is made up of "clauses," all of which must be satisfied simultaneously. Each clause involves three boolean variables (variables with values "true" or "false"), which can be represented by integers $m_{c}=0,1$. In 1-in-3SAT, a clause is satisfied if and only if exactly one of the variables appearing in the clause is true (as opposed to 3SAT, in which at least one must be true) and no boolean negation is necessary. Note that, in terms of integer variables $m_{c}$, a 1-in-3SAT clause containing variables $m_{i}, m_{j}$, and $m_{k}$ can be expressed as

$$
\begin{aligned}
& 1 \leq m_{i}+m_{j}+m_{k} \leq 1, \\
& 0 \leq m_{i}, m_{j}, m_{k} \leq 1 .
\end{aligned}
$$

If the matrices appearing in conditions (i) to (iii) were diagonal, condition (iii) would give us a concise way of writing the coefficients and constants of a set of inequalities such as Eq. (6) in the diagonal elements. However, the problem we are facing here is significantly more challenging: Diagonal matrices will never satisfy condi- 
tions (i) and (ii), and the matrices $L_{0}$ and $A^{(c)}$ cannot be chosen independently, since they are determined by the eigenvectors and eigenvalues of a single matrix $E$.

These substantial obstacles can be overcome, however. The key step in encoding the above boolean constraints in a quantum Liouvillian is to restrict our attention to matrices $L_{0}$ and $A^{(c)}$ with the following special forms:

$$
\begin{gathered}
L_{0}=2 \pi \sum_{i, j} Q_{i, j}|i, i\rangle\left\langle j, j\left|+2 \pi \sum_{i \neq j} P_{i, j}\right| i, j\right\rangle\langle i, j|, \\
A^{(c)}=2 \pi \sum_{i \neq j} B_{i, j}^{(c)}|i, i\rangle\langle j, j|,
\end{gathered}
$$

with coefficient matrices

$$
\begin{aligned}
Q= & \sum_{r} v_{r} v_{r}^{T} \otimes\left(\begin{array}{ll}
1 & 1 \\
1 & 1
\end{array}\right) \otimes\left(\begin{array}{cc}
k+\lambda_{r} & \lambda_{r} \\
\lambda_{r} & k+\lambda_{r}
\end{array}\right) \\
& +\sum_{c} v_{c} v_{c}^{T} \otimes\left(\begin{array}{cc}
1 & -1 \\
-1 & 1
\end{array}\right) \otimes\left(\begin{array}{cc}
k & -\frac{1}{3} \\
\frac{1}{3} & k
\end{array}\right) \\
& +\sum_{c^{\prime}} v_{c^{\prime}} v_{c^{\prime}}^{T} \otimes\left(\begin{array}{cc}
1 & -1 \\
-1 & 1
\end{array}\right) \otimes\left(\begin{array}{ll}
k & 0 \\
0 & k
\end{array}\right), \\
& B^{(c)}=v_{c} v_{c}^{T} \otimes\left(\begin{array}{cc}
1 & -1 \\
-1 & 1
\end{array}\right) \otimes\left(\begin{array}{cc}
0 & 1 \\
-1 & 0
\end{array}\right) .
\end{aligned}
$$

The sets of real vectors $\left\{v_{r}\right\}$ and $\left\{v_{c}, v_{c^{\prime}}\right\}$ should each form an orthogonal basis, and the parameters $k, \lambda_{r}$, and $P_{i, j}$ are also real. The advantage of this restriction is that the action of the $\Gamma$ operation on matrices of this form is somewhat easier to analyze, as can readily be seen from its definition [given in condition (i), above].

It is a simple matter to verify that the eigenvalues and eigenvectors of $L_{0}$ and $B^{(c)}$ do indeed parametrize the logarithms of a matrix $E$, and that the Hermiticity and normalization conditions, (i) and (ii), necessary for $L$ to be a valid quantum Liouvillian are indeed satisfied by the forms given in Eqs. (7)-(10), as long as $w^{T} Q=0$ and $\operatorname{diag}(P)^{\Gamma}$ is Hermitian [where for $d$-dimensional $Q, w=$ $(1,1, \ldots, 1)^{T} / \sqrt{d}$, and $\operatorname{diag}(P)$ denotes the $d^{2}$-dimensional matrix with $P_{i, j}$ down its main diagonal]. Furthermore, the $\mathrm{CCP}$ condition (iii) reduces for this special form to the pair of conditions:

$$
\begin{aligned}
& \sum_{c} B_{i, j}^{(c)} m_{c}+Q_{i, j} \geq 0 \quad i \neq j, \\
& \left(\mathbb{1}-w w^{T}\right)(\operatorname{diag} Q+\operatorname{offdg} P)\left(\mathbb{1}-w w^{T}\right) \geq 0,
\end{aligned}
$$

where $M=(\operatorname{diag} Q+\operatorname{offdg} P)$ denotes the $d$-dimensional matrix with diagonal elements $M_{i, i}=Q_{i, i}$ and off-diagonal elements $M_{i \neq j}=P_{i, j}$.

We now encode the coefficients of the 1-in-3SAT problem from Eq. (6) into the elements of $v_{c}$. For each clause in Eq. (6a), write a " 1 " in a new element of $v_{i}, v_{j}$, and $v_{k}$, and a " 0 " in the corresponding element of all other $v_{c}$ 's.
For each $v_{c}$, write a 1 in a new element of the vector, and write a 0 in the corresponding element of all the other $v_{c}$ 's (these elements will be used to restrict each $m_{c}$ to the values 0 or 1 ). Finally, extend the vectors so that they are mutually orthogonal and have the same length, which can always be done. One can now verify directly that, by choosing appropriate $v_{r}$, Eq. (6) is equivalent to the 1-in3SAT inequalities of Eq. (11b). Furthermore, conditions (i) and (ii) are always satisfied. (See $[20,21]$ for more detail.) Thus, we have succeeded in encoding 1-in-3SAT into the Liouvillian problem. As the latter is equivalent to the Markovianity problem, this proves that the Markovianity problem is itself NP-hard. This construction easily generalizes to the original question of finding which dynamical equations (if any) could have generated a given set of snapshots [20,21]: Any method of finding dynamical equations consistent with the data would obviously also answer the question of whether these exist, allowing us to solve all NP problems.

Note that, on the positive side, by carrying out a bruteforce search for solutions of the corresponding satisfiability problem [in the case considered above, this is 1-in3SAT, but more generally it is an integer semidefinite constraint problem defined by conditions (i) to (iii), which is obviously in NP], we immediately obtain an algorithm for extracting dynamical equations from measurement data that is guaranteed to give the correct answer. Although such an algorithm will not work in practice even for moderately complex systems, the NP hardness proves that we cannot hope for an efficient algorithm (unless $\mathrm{P}=\mathrm{NP}$ ). And it can be applied to systems with few degrees of freedom, making it immediately applicable at least to many current quantum experiments.

What of the classical setting? The classical analogue of the Markovianity problem is the so-called embedding problem for stochastic matrices, originally posed in 1937 [1]. Despite considerable effort [22] the general problem has, however, remained open until now [23]. Strictly speaking, the quantum result does not directly imply anything about the classical problem. Nevertheless, the arguments we have given in the more complicated quantum setting can straightforwardly be adapted to the classical embedding problem $[20,21]$, proving that this is NP hard, too. (See $[20,21]$ for details.)

Discussion.-On the one hand, this work leads to a rigorous algorithm for extracting the underlying dynamical equations from experimental data. For systems with few effective degrees of freedom, as encountered, for example, in all quantum tomography experiments to date [6-10], this gives the first practical and provably correct algorithm for this key task. For systems with many degrees of freedom, the algorithm is necessarily inefficient, with a run time that scales exponentially. But our complexity-theoretic NP-hardness results show that we cannot hope for a polynomial-time algorithm. Note also that the hardness 
cannot be attributed to allowing high-energy processes in the dynamics (high branches of the logarithm), as the reduction from the 1-in-3SAT problem only needs lowenergy dynamics ( $m$ is restricted to 0 or 1 ).

On the other hand, our results also prove that for general systems, deducing the underlying dynamical equations from experimental data is computationally intractable, unless one can show that $\mathrm{P}=\mathrm{NP}$. This hardness result is true whether the system is quantum or classical, and regardless of how much experimental data we gather about the system. These results also imply that various closely related problems, such as finding the dynamical equation that best approximates the data, or testing a dynamical model against experimental data, are also intractable in general, as any method of solving these problems could easily be used to solve the original problem.

Experience would seem to suggest that, while general classical and quantum dynamical equations may be impossible to deduce from experimental data, the dynamics that we actually encounter are typically much easier to analyze. Our results pose the interesting question of why this should be, and whether there is some general physical principle that rules out intractable dynamics.

The authors would like to thank J. I. Cirac, A. Winter, C. Goldschmidt, and J. Martin for valuable discussions. This work has been supported by the Leverhulme Trust, by the EU (QAP, QESSENCE, MINOS, COMPAS, COQUIT, QUEVADIS), by Spanish Grants No. QUITEMAD, No. I-MATH, and No. MTM2008-01366, by the EURYI, the BMBF (QuOReP), and the Danish Research Council (FNU).

[1] G. Elfving, Acta Soc. Sci. Fennicae n. Ser. A 2, 1 (1937).

[2] S. Cook, www.claymath.org/millennium/P_vs_NP/pvsnp .pdf (2000).
[3] L. Ljung, System Identification: Theory for the User (Prentice Hall, Upper Saddle River, NJ, 1999).

[4] K. Soutwell Nature (London) 453, 1003 (2008).

[5] M. A. Nielsen and I. L. Chuang, Quantum Computation and Quantum Information (Cambridge University Press, Cambridge, 2000).

[6] M. Nielsen, E. Knill, and R. Laflamme, Nature (London) 396, 52 (1998).

[7] N. Boulant, T. F. Havel, M. A. Pravia, and D. G. Cory, Phys. Rev. A 67, 042322 (2003).

[8] M. Riebe et al., Phys. Rev. Lett. 97, 220407 (2006).

[9] J. L. O'Brien et al., Phys. Rev. Lett. 93, 080502 (2004).

[10] M. Howard et al., New J. Phys. 8, 33 (2006).

[11] J. R. Norris, Markov Chains (Cambridge University Press, Cambridge, 1997).

[12] G. Lindblad, Commun. Math. Phys. 48, 119 (1976).

[13] H. J. Carmichael, Statistical Methods in Quantum Optics (Springer, New York, 2003), Vol. 1.

[14] V. Gorini, A. Kossakowski, and E. C. G. Sudarshan, J. Math. Phys. (N.Y.) 17, 821 (1976).

[15] M. Howard et al., New J. Phys. 8, 33 (2006).

[16] M. M. Wolf and J. I. Cirac, Commun. Math. Phys. 279, 147 (2008).

[17] M. M. Wolf, J. Eisert, T. S. Cubitt, and J. I. Cirac, Phys. Rev. Lett. 101, 150402 (2008).

[18] H.-P. Breuer, Phys. Rev. A 75, 022103 (2007).

[19] M. R. Garey and D.S. Johnson, Computers and Intractability: A Guide to the Theory of NPCompleteness (W. H. Freeman, New York, 1979).

[20] See Supplemental Material at http://link.aps.org/ supplemental/10.1103/PhysRevLett.108.120503 for more details of the proof.

[21] T. S. Cubitt, J. Eisert, and M. M. Wolf, Commun. Math. Phys. 310, 383 (2012).

[22] J.F. C. Kingman, Z. Wahrsch. Verw. Geb. 1, 14 (1962).

[23] A. Mukherjea, in Matrix Theory and Applications, edited by C.R. Johnson (American Mathematical Society, Providence, RI, 1990). 